\title{
Percolation in deposits for competitive models in $(1+1)$-dimensions
}

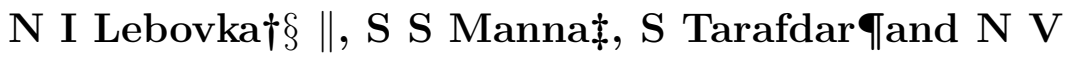 \\ Vygornitskii $\dagger$ \\ $\dagger$ Institute of Biocolloid Chemistry NASU, 2, bulv. Vernadskogo, Kyiv, 03142, \\ Ukraine \\ $\ddagger$ Satyendra Nath Bose National Centre for Basic Sciences Block-JD, Sector-III, Salt \\ Lake, Kolkata-700098, India \\ ฯ Condensed Matter Physics Research Centre, Physics Department, Jadavpur \\ University, Kolkata 700032, India
}

\begin{abstract}
The percolation behaviour during the deposit formation, when the spanning cluster was formed in the substrate plane, was studied. Two competitive or mixed models of surface layer formation were considered in $(1+1)$-dimensional geometry. These models are based on the combination of ballistic deposition (BD) and random deposition (RD) models or BD and Family deposition (FD) models. Numerically we find, that for pure RD, FD or BD models the mean height of the percolation deposit $\bar{h}$ grows with the substrate length $L$ according to the generalized logarithmic law $\bar{h} \propto(\ln (L))^{\gamma}$, where $\gamma=1.0(\mathrm{RD}), \gamma=0.88 \pm 0.020$ (FD) and $\gamma=1.52 \pm 0.020$ (BD). For BD model, the scaling law between deposit density $p$ and its mean height $\bar{h}$ at the point of percolation of type $p-p_{\infty} \propto \bar{h}^{-1 / \nu_{h}}$ are observed, where $\nu_{h}=1.74 \pm 0.02$ is a scaling coefficient. For competitive models the crossover, corresponding to the RD or FD -like behaviour at small $L$ and the BD-like behaviour at large $L$ are observed.

PACS numbers: 64.60.Ak, 68.00.00, 72.60.+g, 81.15.Aa, 89.75.Da
\end{abstract}

Submitted to: Institute of Physics Publishing

$\S$ To whom correspondence should be addressed.

|| E-mail: lebovka@roller.ukma.kiev.ua 


\section{Introduction}

Formation of thin films in the process of deposition and aggregation of particles on a substrate has received a considerable theoretical and experimental attention in recent years [1, 2]. This problem is very important also from the practical point of view for production of thin-film devices and conducting composite films with certain specified electrical [3, 4], magnetic, transport [5] and colloidal properties [6, 7]. There also exist large interest in studying morphology of deposits [8, 9, 10, their fractal [11, 12] and percolating properties [13, 14, 15, 16].

Among the most popular models for simulation of deposits formation there are models of random deposition (RD), random deposition with surface relaxation or Family deposition (FD) and ballistic deposition (BD) and different their variants [1, 2]. In these models, the particles are rigid and cannot overlap and these models describe growth processes far from equilibrium. In RD model the particles deposit without sticking and it means the presence of the short-range repulsion. In FD model the particles can relax to a lower nearest neighbor position. In BD model the particles stick at a point of the first contact and it means the presence of the short-range attraction. Recently there were proposed a number of mixed or competitive models, which are based on consideration of deposition from different kinds of particles [17, 18.

The percolation phenomena in growing simulated films were previously analyzed for different models of deposits on two-dimensional substrate [19, 20, 21, 22, 23]. The present paper addresses the percolation behaviour for different competitive $(1+1)$ dimensional lattice models of deposit formation on a line substrate.

The paper is organized as follow. The model is described in section 2. In section 3 , the scaling behaviour of deposit height at percolating point and its density are discussed. Concluding remarks are presented in section 4

\section{Model}

In our (1+1)-dimensional competitive models there exist two kinds of particles, following $\mathrm{BD}$ rules and $\mathrm{RD}$ or $\mathrm{FD}$ rules. We call these models as $\mathrm{BD}_{1-s} \mathrm{RD}_{s}$ or $\mathrm{BD}_{1-s} \mathrm{FD}_{s}$, respectively, where $s$ is a fraction of RD or FD particles. Each particle falls along vertical direction, until it reaches the interface. Particles get deposited one after another and fixed in the sites of square lattice according to the deposition rules.

We stop the growth process when the spanning cluster forms for the first time in the substrate plane and this point is easily checked by a Hoshen-Kopelman algorithm 24. The percolation in deposits differs from usual random percolation [25] and has a correlated character, because the sites of lattice get filled dynamically during the growth of deposit.

The mean height of deposit at the percolation point is calculated as $\bar{h}=\Sigma_{i} h_{i} / L$, where $L$ is the substrate length. The time $t$ is counted as the number of deposited particles $N=L$. The deposit density $p$ is calculated as the ratio of the number of 


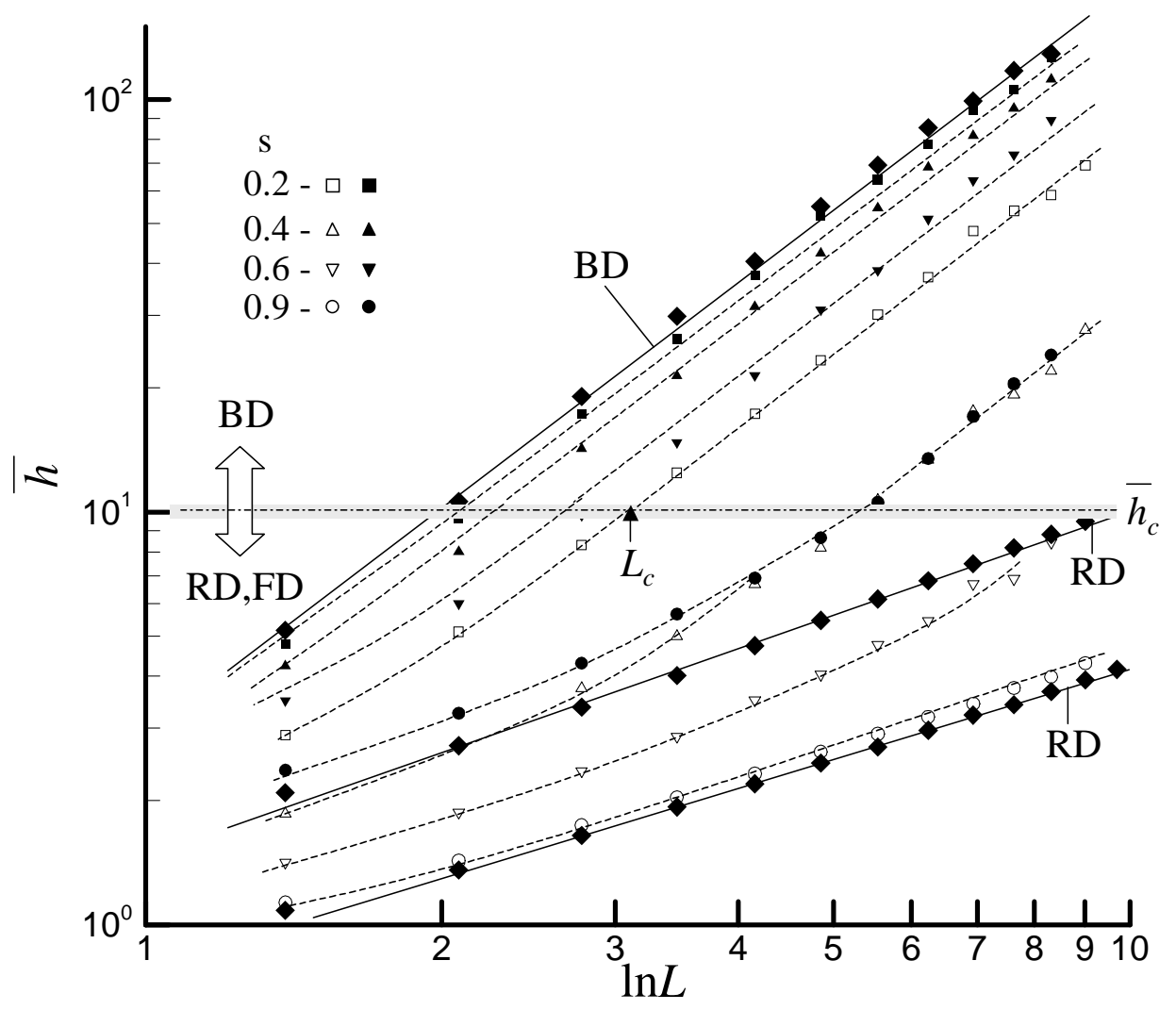

Figure 1. Mean height of deposit at the percolation point $\bar{h}$ versus substrate length $L$ for $\mathrm{BD}_{1-s} \mathrm{FD}_{s}$ (open symbols) and $\mathrm{BD}_{1-s} \mathrm{RD}_{s}$ (filled symbols) models at different $s$. Here the crossover line $\bar{h} \approx 10$ between regimes with different $\bar{h}$ versus $L$ behaviour is shown. The data error is of the order of data symbol size. The dashed lines serve as a guide to the eye. Both of $\bar{h}$ and $L$ are in lattice units.

particles and the deposit volume $p=N /(L \bar{h})=t / \bar{h}$.

The substrate size $L$ was varied from $2^{2}$ to $2^{17}$ and the periodical boundary conditions were used in the deposition rules along the substrate direction. Results were averaged over 100-5000 different runs, depending on the size of the lattice and required precision.

\section{Results and discussion}

In figure 1 we show the numerically determined mean height of the deposit at the percolation point $\bar{h}$ versus the substrate length $L$ for competitive models $\mathrm{BD}_{1-s} \mathrm{RD}_{s}$ and $\mathrm{BD}_{1-s} \mathrm{FD}_{s}$ at various values of $s$. A remarkable feature of data presented in figure 1 is that for for pure $\mathrm{RD}(s=1), \mathrm{FD}(s=1)$ or $\mathrm{BD}(s=0)$ models, the value of $h$ grows with the substrate length $L$ according to the generalized logarithmic law:

$$
h \propto(\ln L)^{\gamma},
$$

where $\gamma$ is an exponent that is $\gamma_{R D}=1.0, \gamma_{F D}=0.88 \pm 0.02$ and $\gamma_{B D}=1.52 \pm 0.02$ for RD, FD and BD models, respectively. 


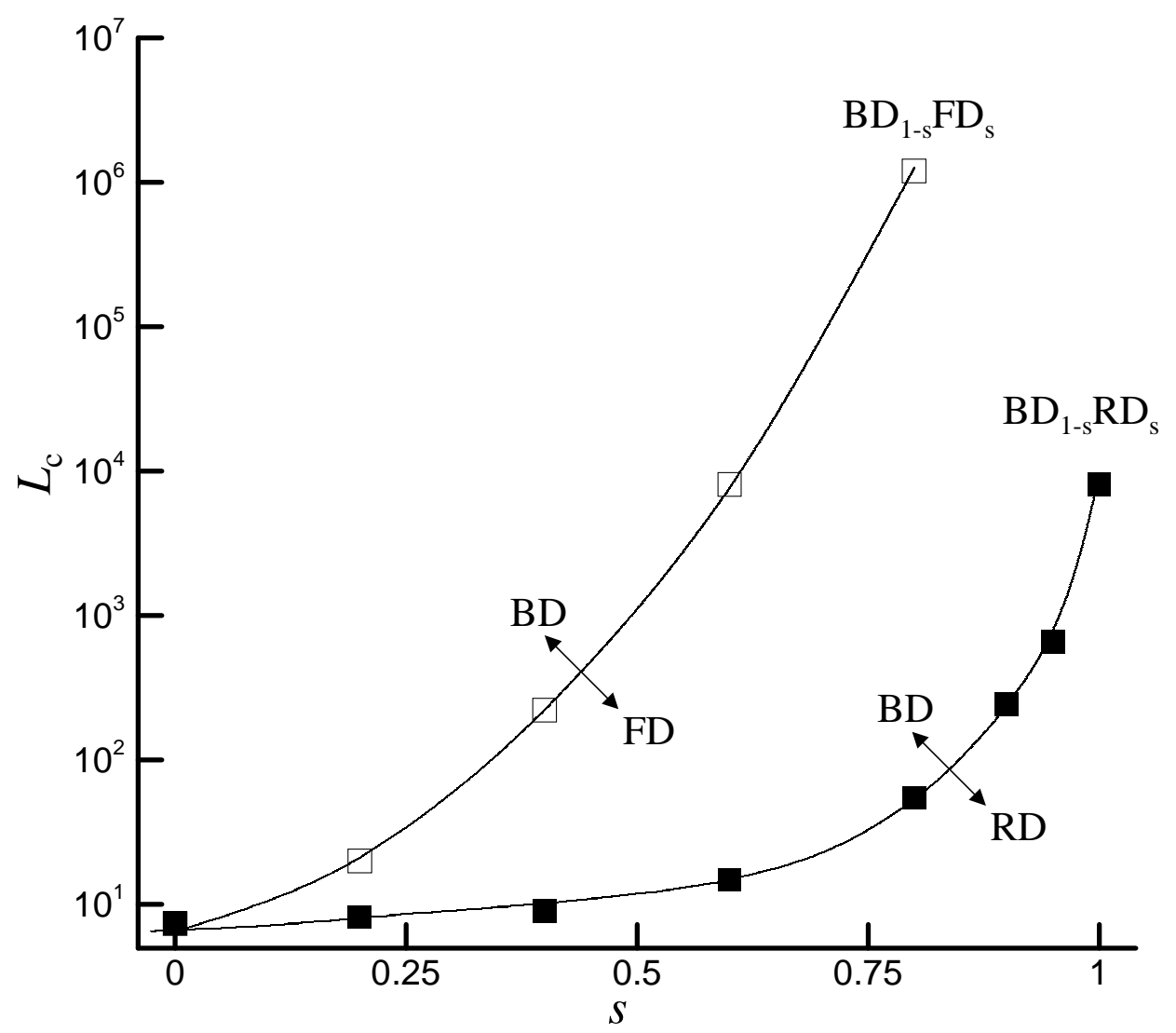

Figure 2. Crossover length $L_{c}$ versus $s$ for $\mathrm{BD}_{1-s} \mathrm{FD}_{s}$ and $\mathrm{BD}_{1-s} \mathrm{RD}_{s}$ models.

For RD model the logarithmic law $\bar{h}=\ln L$ can be easily justified. In the RD model, every column of deposit grows independently, and in the limit of $L \gg 1$ the distribution function of heights follows the Poisson law:

$$
P(h)=\left(e^{-\bar{h}}\right)\left(\bar{h}^{h}\right) / h !
$$

Therefore, the probability to find of an empty column $(h=0)$ is equal to $P(0)=$ $e^{-\bar{h}}$. In the percolation point the last empty column gets filled, and $P(0)=e^{-\bar{h}}=1 / L$. So, we obtain for RD model exactly

$$
\bar{h}=\ln L,
$$

and therefore $\gamma=1$ in (11) for that model.

For pure $\mathrm{BD}$ and FD model, there exist correlations between the columns and exact relation can not be obtained by this simple way. For both RD and FD models, the deposits are non-porous and $p=1$ at any time during the deposit formation. Moreover, in the percolation point at the same $L$, the mean height of the deposit is less for FD model than for RD model, i.e., $\bar{h}_{F D}<\bar{h}_{R D}$. For BD model, the deposit is porous and, therefore, $\bar{h}_{R D}<\bar{h}_{B D}$ at the same $L$. So, it is reasonable to expect that $\gamma_{F D}<\gamma_{R D}<\gamma_{B D}$ and it is completely in accordance with numerical data presented in figure 1

For competitive $\mathrm{BD}_{1-s} \mathrm{FD}_{s}$ and $\mathrm{BD}{ }_{1-s} \mathrm{RD}_{s}$ models, the data follow the generalized logarithmic law (11) only at large values of $\bar{h}$ and systematic deviations from 


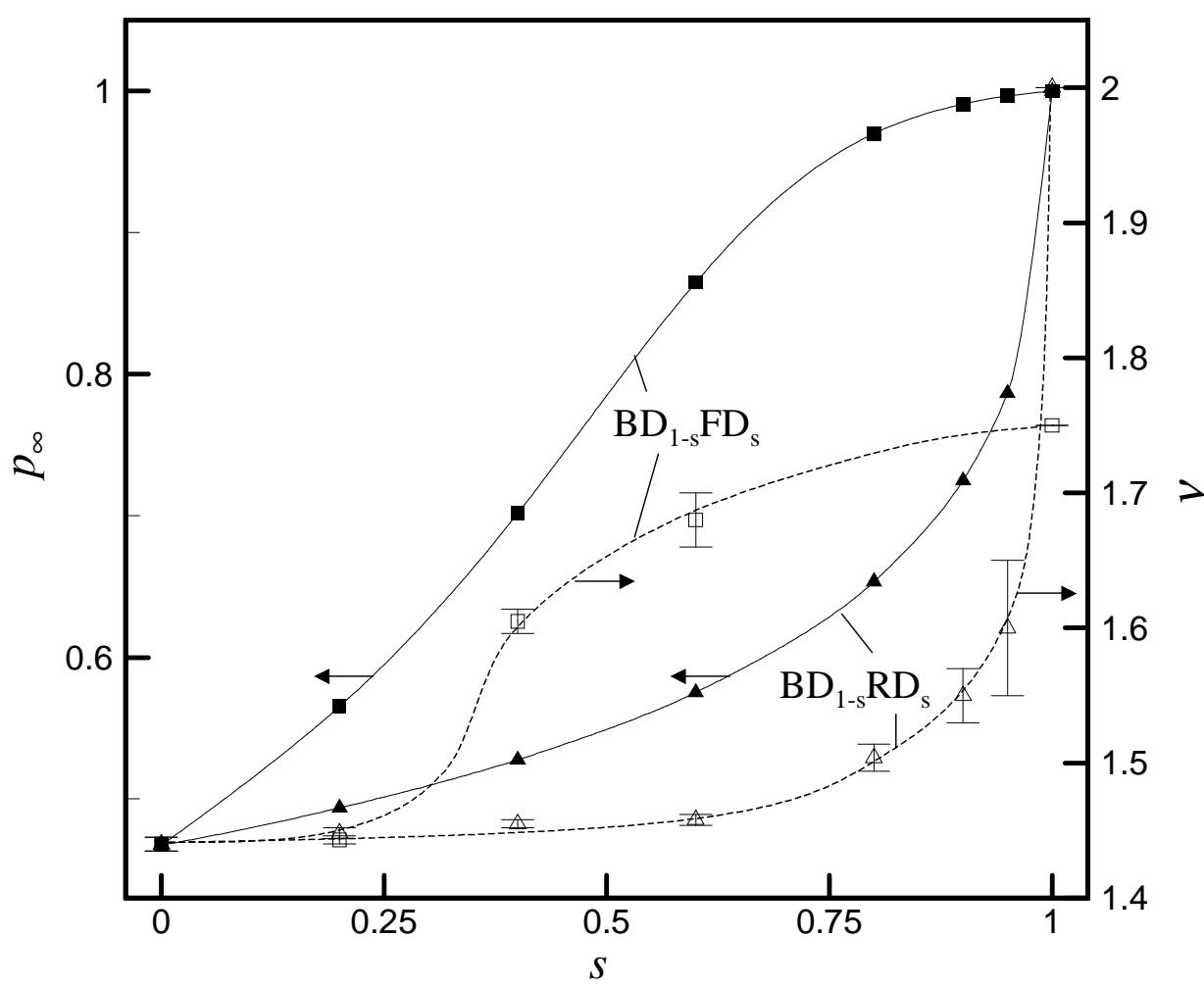

Figure 3. Plots of density of deposit $p_{\infty}$ and scaling exponent $\nu$ in (4) versus $s$ for $\mathrm{BD}_{1-s} \mathrm{FD}_{s}$ and $\mathrm{BD}_{1-s} \mathrm{RD}_{s}$ models. In cases when it is not show directly the data error is of order of data symbol size. The lines serve as a guide to the eye.

this law are observed $\bar{h} \lesssim \bar{h}_{c}$, where $\bar{h}_{c} \approx 10$. We attribute the origin of this behaviour to the known random-like nature of the deposition process at small $\bar{h}$, when there are no strong correlations between growth columns in the deposit, and the choice of the boundary value $h_{c}$ is rather approximate [18].

So, for the competitive model with a given $s$, the random-like processes can control the $\bar{h}$ versus $L$ behaviour at small $L$ and there exists a strong crossover to the BD-like behaviour at $L>L_{c}$, where $L_{c}$ is the crossover length. We have estimated the value of $L_{c}$ from the intersection point of $\bar{h}(L)$ line with the horizontal line $\bar{h}=h_{c} \approx 10$. The crossover length $L_{c}$ is always smaller for $\mathrm{BD}_{1-s} \mathrm{RD}_{s}$ model than $L_{c}$ for $\mathrm{BD}_{1-s} \mathrm{FD}_{s}$ model at the same $s$ (see figure 2).

It is interesting to check for existence of some scaling between the value of the deposit density $p$ and its height $\bar{h}$ in the percolation point. In order to do this analysis correctly, we need for a limiting value of a deposit density $p_{\infty}$ for infinitely large systems. We have analyzed the $p$ versus $L$ dependencies for the systems of size $L \times \bar{h}$, with $\bar{h}=L$ and have found that all the data can be fitted with the following scaling relation:

$$
p-p_{\infty} \propto L^{-1 / \nu},
$$

where $p_{\infty}$ is the deposit density in the limit of $L \rightarrow \infty$, and $\nu$ is an exponent.

For BD model $p_{\infty, B D}=0.4673$ (this result is in accordance with [26]) and $\nu_{B D}=1.44 \pm 0.01$, and $p_{\infty}$ and $\nu$ increase with $s$ increase for both $\mathrm{BD}_{1-s} \mathrm{FD} s$ and $\mathrm{BD}$ 


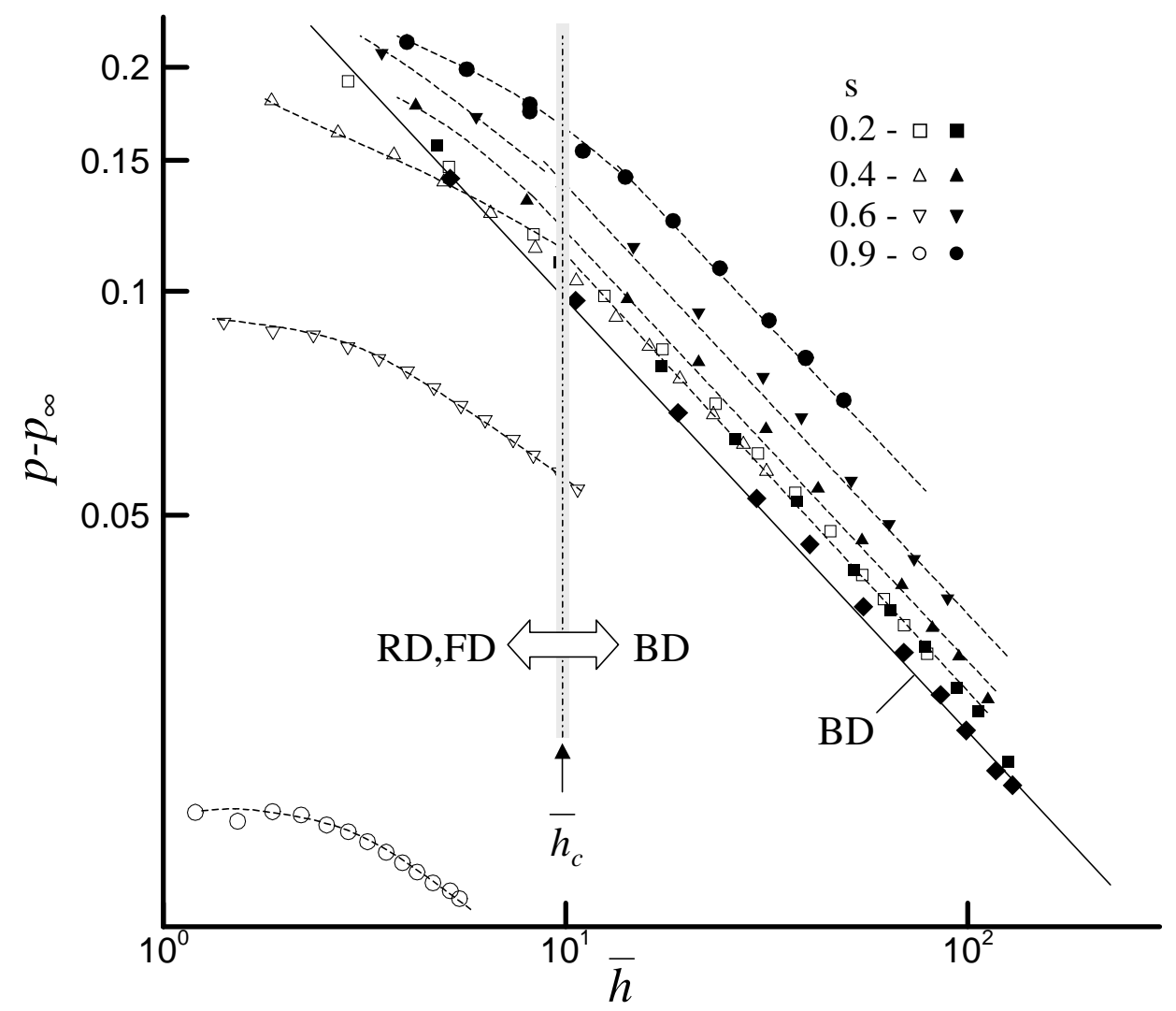

Figure 4. Log-log plot of $p-p_{\infty}$ versus $\bar{h}$ (lattice units) for $\mathrm{BD}_{1-s} \mathrm{FD}{ }_{s}$ (open symbols) and $\mathrm{BD}_{1-s} \mathrm{RD}_{s}$ (filled symbols) models at different $s$. Here the crossover line $\bar{h} \approx 10$ is shown. The data error is of order of data symbol size. The dashed lines serve as a guide to the eye. The solid line corresponds to best fit of (6) to the data for BD model(filled diamonds) with scaling exponent $\nu_{h}=1.74 \pm 0.02$.

${ }_{1-s} \mathrm{RD}_{s}$ models (figure 3 ).

We can estimate the values of $\nu_{R D}$ and $\nu_{F D}$ from the following reasonings. The number of the deposited particles $N$ in the system of size $L \times \bar{h}=L \times L$ with the rough upper interface can be estimated as $N=L(L-a \omega)$, where $a$ is some constant and $\omega$ is the interface width. The value of $\omega$ scales at small times $t=\bar{h}$ as $\omega \propto t^{\beta}$, where $\beta$ is the growth exponent which is equal to $1 / 2$ for $\mathrm{RD}$ model and to $1 / 4$ for FD model 2]. For compact deposits $p=1, t=\bar{h}$ and, so, $\omega \propto \bar{h}^{\beta}=L^{\beta}$. For both RD and FD models, the height of deposit in our systems $\bar{h}=L$ corresponds to the the small-time regime, because the time of transition to the saturation regime is $t_{x}=\infty$ for the $\mathrm{RD}$ model and of order $t_{x} \approx L^{2}$ for the FD model. So, the density of the deposit at $s \rightarrow 1$ can be estimated as:

$$
p=N / L^{2}=L(L-a \omega) / L^{2}=1-a L^{-1 / \nu},
$$

where $\nu=1 /(1-\beta)$, and $\nu_{R D}=2$ and $\nu_{R D}=4 / 3$ for RD and FD models, respectively. 
Figure 4 shows the log-log presentation of $p-p_{\infty}$ versus $\bar{h}$ for the $\mathrm{BD}_{1-s} \mathrm{FD} s$ and $\mathrm{BD}_{1-s} \mathrm{RD}_{s}$ models. The evident scaling of type

$$
p-p_{\infty} \propto \bar{h}^{-1 / \nu_{h}}
$$

is observed outside the random-like deposition regime at $\bar{h} \gtrsim 10$ for both $\mathrm{BD}_{1-s} \mathrm{FD}{ }_{s}$ and $\mathrm{BD}_{1-s} \mathrm{RD}_{s}$ models. At $\bar{h} \gtrsim 10$, the scaling exponent at different $s$ was approximately same as for pure BD model $\nu_{h}=1.74 \pm 0.02$.

\section{Conclusions}

In summary, we have investigated the percolation in the direction parallel to the surface for competitive $(1+1)$ dimensional models of deposition layer formation. The height of the percolating deposit $\bar{h}$ shows the continuous growth with increase of the substrate length $L$, and percolation is absent in the limit of infinite systems $L \rightarrow \infty$. This behaviour is different from that observed for $(2+1)$ dimensional model, where a percolating deposit with finite height was formed 23]. The competitive models always show a crossover to the BD-like deposition behaviour at the limit of very large $L$.

\section{Acknowledgements}

NL and NV thanks the NASU for partial financial support under the projects No. 2.16.1.4 (0102V007058) and 2.16.2.1(0102V007048).

\section{References}

[1] Family F and Vicsek T 1991 Dynamics of Fractal Surfaces (Singapore: World Scientific)

[2] Barabási A -L and Stanley H E 1995 Fractal Concepts in Surface Growth (Cambridge: Cambridge University Press)

[3] Ivashchenko A I, Kerner Ia I, Kiosse G A and Maronchuk I Yu 1997 Thin Solid Films 303 292-294

[4] Texter J and Lelental M 1999 Langmuir 15 654-661

[5] Pohorilyi A N, Kravetz A F, Shypil O V, Vovka A Ya, Kima C S and Khanb H R 1999 J. Magn. Magn. Mater. 196-197 43-44

[6] Wang Y. and Anderson C 1999 Macromolecules 32 6172-6179

[7] Roldughin V I and Vysotskii V V 2000 Progress in Organic Coatings 39 81-100

[8] Nainville I, Lemarchand A and Badiali J P 1996 Electrochimica Acta 41 1855-1862

[9] Tarafdar S and Roy S 1998 Physica B254 28-36

[10] Dasgupta R, Roy S and Tarafdar S 2000 Physica A275 22-32

[11] Voss R F, Laibowitz R B and Alessandrini E I 1981 Phys. Rev. Lett. 49 1441-1444

[12] Dobierzewska-Mozrzymas E, Biegaski P, Pieciul E and Wojcik J 1997 Vacuum 48 293-297

[13] Kapitulnik A and Deutscher G 1982 Phys. Rev. Lett. 49 1444-1448

[14] Kusy A 1997 Physica B240 226-241

[15] Yamamuro S, Sumiyama K, Hihara T and Suzuki K 1999 J. Phys.: Condens. Matter 11, 3247-3257

[16] Gadenne M, Schneegans O, Houze F, Chretien P, Desmarest C, Sztern J and Gadenne P 2000 Physica A279 94-97

[17] El-Nashar H F and Cerdeira H A 2000 Physica B283 6-12

[18] Horowitz C M Monetti R A and Albano E V 2001 Phys. Rev. E63 066131

[19] Silverman B D and Platt D E 1994 Phys. Rev. E49 1028-1039 
[20] Jensen P, Barabasi A -L, Larralde H, Havlin S and Stanley H E 1995 Chaos, Solitons and Fractals 6 227-331

[21] Family F 1999 Physica A266 173-185

[22] Sotta P and Long D 2003 Preprint Cond-Mat 0301120

[23] Lebovka N I, Manna S S, Tarafdar S and Teslenko N 2002 Phys. Rev. E66, 066134

[24] Hoshen J and Kopelman R 1976 Phys. Rev. B14 34383445

[25] Stauffer D and Aharony A 1994 Introduction to Percolation Theory (London: Taylor and Francis)

[26] Krug J and Meakin P 1991 Phys. Rev. A43 900-919 